\title{
From Rough Surface to Peri-implantitis, Hybrid Implants and Subcrustal Implant Placement: A Mini Review of Last Decade Research and Clinical Findings in Implant Dentistry
}

ISSN: 2637-7764

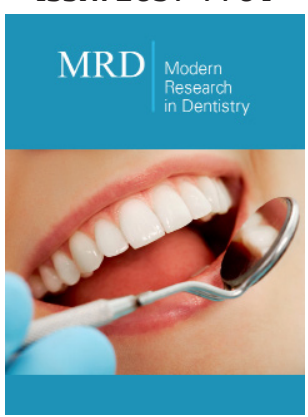

*Corresponding author: Andrze Wojtowicz,Department of Oral Surgery, Warsaw Medical University, Polish Association in Implantology

Submission: 鯆 November 23, 2020

Published: 㭗 December 04, 2020

Volume 5 - Issue 5

How to cite this article: Witold Tomkiewicz and Andrzej Wojtowicz. From Rough Surface to Peri-implantitis, Hybrid Implants and Subcrustal Implant Placement: A Mini Review of Last Decade Research and Clinical Findings in Implant Dentistry. Mod Res Dent. 5(5). MRD. 000623. 2020.

DOI: $10.31031 /$ MRD.2020.05.000623

Copyright@ Andrzej Wojtowicz, This article is distributed under the terms of the Creative Commons Attribution 4.0 International License, which permits unrestricted use and redistribution provided that the original author and source are credited.

\section{Witold Tomkiewicz and Andrzej Wojtowicz*}

Department of Oral Surgery, Warsaw Medical Univeristy, Polish Association in Implantology

\begin{abstract}
Continuous effort to safely apply occlusal load immediately, or as early as possible, led thru improved titanium surfaces to give more rapid osseointegration on a bigger implant surface (BIC). Around year 2000 increasing implant roughness became a key with a wish to inoculate active, bone growing factors in it. Surprisingly after few years a totally new phenomenon of progressive bone loss around implants was observed and coined 'peri-implantitis' as a parallel to 'periodontitis'. Similar pathogens became a suspect however exposed rough surface is the main trigger. Retrospective studies of machined implants led to hybrid implant design on one hand and recommendations of subcrestal placement of fully rough implants on the other(Figure 1). Additionally, more emphasis has been put on surgical technique and timing.
\end{abstract}

Implant as a Foreign Body

Albrektsson et al. [1] Already in 2013 note that making a direct parallel between marginal bone loss around dental implants in peri-implantitis and bone loss around teeth in periodontitis is a wrong track. It is therefore not a biofilm-mediated infectious processes, but tissue reactions coupled to the foreign body response. The initial foreign body response to an implant can be modulated (aggravated) by implant hardware characteristics, surgical technique and patients reparative and compliance capacity. This marginal bone loss, also related to as 'remodeling' process, was well known and described as normal or physiological in the literature by Branemark implants authors as the resorption to the $1^{\text {st }}$ thread during the $1^{\text {st }}$ year after abutment placement. Then the bone level became stable. Once achieved this dynamic equilibrium is not a given' forever but a subject to many dis-balancing risk factors at any given point in time.

\section{Bacteria Comes Secondary}

Further Albrektsson et al. Indicate that once severe marginal bone loss is developed, a secondary biofilm-mediated infection may follow as a complication to the already established bone loss. The extend of this infection and subsequent bone loss was related to the implant roughness by Albouy, Abrahamsson and Berglundh [2-5] in their experimental study of ligature induced peri-implantitis in dogs.

\section{Machined Surface Limits Biofilm Formation and May Stop Periimplantitis}

The researchers conclude that the amount of bone loss that occurred during the plaque accumulation period after ligature removal was significantly larger at implants with a rough (TiUnite surface) than at implants with a machined (turned) surface. The histological analysis revealed that the vertical dimensions of the lesion, the pocket epithelium and the apical extension of the biofilm were also significantly larger at rough implants. Another words, when the ligature was removed on machined implants the bone loss was stopped while it continued on the rough surface.

\section{Has this Been Demonstrated Clinically on Patients with Periimplantitis?}

Schwarz et al. [6] have quite extensively presented and published follow ups of periimplantitis treatment where they compared different methods of surface decontamination 
as an add-on to standard surgical treatment of implantoplasty. Although laser decontamination did not prove to be any better than saline solution in their study the overall treatment success of stopping progression of periimplantitis was achieved in over $80 \%$ of cases. The result therefore could only be attributed to standard implantoplasty which is grinding exposed contaminated surface of an implant with a dimond drill on high speed. The roughness of the surface after implantoplasty is very similar to machined surface and also interestingly among the implants treated there were no machined (Branemark) implants but only implants with rough surface.

\section{Hybrid implants}

The risk of rough surface to form biofilm has already been indicated in 1993 by Dennis P Tarnow [7] who already then suggested a hybrid implant with a machined titanium surface in the coronal half and a rough titanium surface in the apical half. 'This will theoretically allow for minimal plaque accumulation at the crest if the implant becomes exposed to the oral environment'. Tarnow's idea for hybrid implants have become materialised after 25 years by Massimo Simion [8] who has also demonstrated that the rough surface is not any better forming bone than 'old' machined surface (Figure 1).
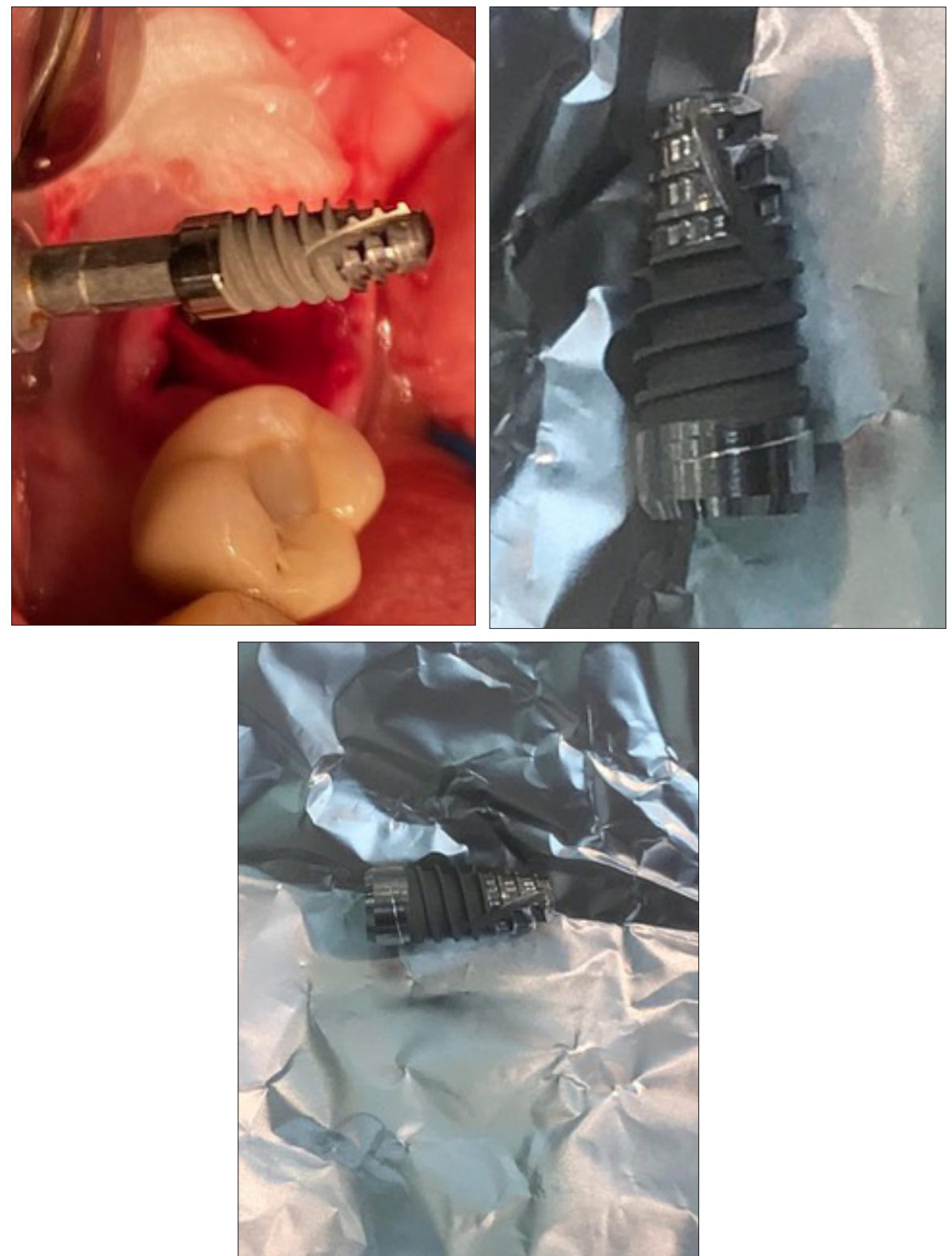

Figure 1: A prototype of a 3-hybrid implant with surfaces dedicated to 3 different tissue compartments. Additionally, implant coated with double covalent layer of hyaluronic acid and its geometry modified with vertical grove to shred more bone chips during insertion. 


\section{Machined vs Rough}

Simion et al. [8] conclude that 'the surface roughness may not be a key factor for successful osseointegration of immediately or early loaded implants' and that 'osseointegration follows a similar healing pattern with machined and oxidized implant surfaces. This common healing healing pattern observed by Simion et al. [8] in their histologic study was early bone formation around the bone chips which appear to be foci of osteogenesis. They observed that the bone began to grow on an implant surface if there was a bone chip there irrespective of the surface: machined or rough.

\section{Surgical Technique}

Considering above findings and high incidence of periimplantitis reported in the last decade-surgical technique changed towards limiting the risk of rough implant surface exposure. This includes subcrestal placement of fully rough implants as well as minimally invasive approach with one-time abutment concept. Also, palatal implant positioning and preventive tissue augmentations are the present measures to limit the risk of periimplantitis.

\section{Conclusion and Considerations of Future Trends}

Hybrid implants open a new perspective of applying surfaces dedicated to different tissue compartments. As machined surface is best safe coronally, rough in the middle may still give convenient friction and primary implant stability. The question remains about the apical portion of an implant that interfaces bone marrow. What would the best surface be there for the stem cells to migrate, spread and differentiate? How soon will we have growth factors spread on the implant surface and will it work as we desire? Some laboratory experiments in the past showed however that even if we inoculated BMP inside the pores of titanium discs the bone would vigorously grow around but not stick to the disc. Pouring however growth factors (rh PDGF-BB) into an implant osteotomy contributed in observable increase in BIC which was presented by Myron Nevins and David Kim, researchers from Harvard School of Dental Medicine. So far we observe that raw and fresh titanium surface, free from air hydrocarbonate contamination, may be most active and therefore some implants stored in sterile saline solution. Another interesting option is surface coating with Hyaluronic Acid [9] double covallent layer knowing that hyaluronic acid is an inter-cellular matrix building compound and therefore may facilitate osseointegration, but this needs to be confirmed best with further histology studies. If we stick to what we know which is that bone chips are foci of new bone formation in the space between an implant and native bonewe shall benefit from it clinically by modifying implant geometry to create more bone chips and also adding bone chips into osteotomy.

\section{References}

1. Albrektsson T, Dahlin C, Jemt T, Sennerby L, Turri A, et al. (2014) Is marginal bone loss around oral implants the result of a provoked foreign body reaction? Clinical Implant Dentistry and Related Research 16(2): 155-165.

2. Albouy JP, Abrahamsson I, Berglundh T (2012) Spontaneous progression of experimental peri-implantitis at implants with different surface characteristics: an experimental study in dogs. J Clin Periodontol 39(2): 182-187.

3. Berglundh T, Gotfredsen K, Zitzmann NU, Lang NP, Lindhe J (2007) Spontaneous progression of ligature induced peri-implantitis at implants with different surface roughness: an experimental study in dogs. Clin Oral Implants Res 18(5): 655-661.

4. Albouy JP, Abrahamsson I, Persson LG, Berglundh T (2011) Implant surface characteristics influence the outcome of treatment of periimplantitis: an experimental study in dogs. J Clin Periodontol 38(1): 5864.

5. Carcuac O, Abrahamsson I, Albouy JP, Linder E, Larsson L, et al. (2013) Experimental periodontitis and peri-implantitis in dogs. Clin Oral Implants Res 24(4): 363-371.

6. Schwarz F, John G, Schmucker A, Sahm N, Becker J (2017) Combined surgical therapy of advanced peri-implantitis evaluating two methods of surface decontamination: a 7-year follow-up observation. J Clin Periodontol 44(3): 337-342.

7. Tarnow DP (1993) Dental implants in periodontal care-Current opinion in periodontology pp. 157-162.

8. Simion M, Benigni M, Hezaimi KA, Kim DM (2015) Early bone formation adjacent to oxidized and machined implant surfaces: a histologic study. Int J Periodontics Restorative Dent 35(1): 9-17.

9. Lupi SM, Baena ARY, Cassinelli C, Iviglia G, Tallarico M, et al. (2019) Covalently-linked hyaluronan versus acid etched titanium dental implants: A crossover RCT in humans. Int J Mol Sci 20(3): 763. 